\title{
Epididymal or Testicular Ultrasonic Findings: Which One is More Reliable for Differentiation of Testicular Torsion from Epididymitis?
}

\author{
Reihaneh Mortazavi, ${ }^{1}$ Seyed Ali Alamdaran, ${ }^{2,}$ Ahmad Mohamadipour, ${ }^{3}$ and Ali Feyzi laein ${ }^{1}$ \\ ${ }^{1}$ Radiology Department, Mashhad University of Medical Sciences, Mashhad, IR Iran \\ ${ }^{2}$ Surgical Oncology Research Center, Mashhad University of Medical Sciences, Mashhad, IR Iran \\ ${ }^{3}$ Pediatric Surgery Department, Mashhad University of Medical Sciences, Mashhad, IR Iran \\ "Corresponding author: Seyed Ali Alamdaran, Surgical Oncology Research Center, Mashhad University of Medical Sciences, Mashhad, IR Iran. Tel: +98-9155112578, Fax: \\ +98-5138414499, E-mail: alamdarana@mums.ac.ir
}

Received 2018 April 19; Accepted 2018 May 07.

\begin{abstract}
Background: Ultrasound is the primary modality for the evaluation of patients with acute scrotum. Accurate exclusion of testicular torsion is prevented from unnecessary surgical exploration.

Objectives: We assessed scrotal changes in pediatric testicular torsion in comparison epidydimits, with purpose to determine more specific points for differentiation testicular torsion from epididymitis.

Methods: During 2011 - 2017 a descriptive case control study was performed in Dr. Sheikh and Akbar Children hospital, Mashhad medical university of science. The 41 pediatric patients with acute scrotum ( 21 cases with testicular torsion and 20 cases with epididymitis) were examined. Eventually, the sonographic findings were analyzed to compare the results.

Results: Testicular and epididymal enlargement, hydrocele, the hyperemia of surrounding tissues and the scrotal skin thickening are observed in both epididymitis and torsion without any significant difference $(\mathrm{P} \geq 0.05)$. Some other findings where observed in both groups with a significant difference $(P \leq 0.05)$ such as changes in echogenicity of testis and epididymis, abnormal testicular axis and spermatic cord changes are observed in both epididymitis and torsion; but they had low sensitivity. The most specific signs of testicular torsion were testicular parenchymal heterogenicity (94\%), testicular flow pattern (94\%), increased echogenicity of epididymis (73\%), heterogenicity of epididymis (84\%), abnormal epididymis location (100\%), mass-like configuration of epididymis (100\%) and epididymis flow pattern (100\%). The most epididymal findings are more specific and sensitive than testicular findings. Conclusions: Avascularity, heterogenicity, displacement and mass-like configuration of epididymis are reliable sonographic findings for differentiation testicular torsion from epididymitis. They have high diagnostic value with sensitivity of $75 \%-100 \%$ and specificity of $84-100 \%$. So, making proper use of them can minimize diagnostic pitfalls.
\end{abstract}

Keywords: Testicular Torsion, Epididymitis, Ultrasound

\section{Background}

Torsion of spermatic cord is a urologic emergency. It is the most important acute scrotal abnormality that needs a fast diagnosis for surgical exploration (1-3). Ultrasound is the primary modality for the evaluation of patients with acute scrotal pathologies $(3,4)$ and testicular torsion can cause changes in grey-scale, color, and spectral Doppler sonography depending on the degree of twist and the duration of symptoms (2). Sonographic findings of testis in these patients vary from normal pattern to spherical enlargement and eventually, atrophy, abnormal axis of affected testis, diffusely hypoechoic echo texture, heterogeneous, focal or multifocal echogenicity due to hemorrhagic infarction, thick mediastinum, and absence or decreased blood flow in the affected testis $(1,2,5,6)$. However, the presence of the color signal in a portion of the testis (usually near or inside the mediastinum) does not exclude spermatic cord torsion (6). Additional findings associated with torsion are scrotal skin thickening more than $5 \mathrm{~mm}$, the presence of coiled spermatic cord, and reactive hydrocele with hyper-vascularity at thickened scrotal tissue surrounding testis $(1,2,6)$.

The similar clinical and ultrasonographic signs are observed in epididymitis, which is the top differential diagnosis for testicular torsion. The most common ultrasonic features of epididymitis include epididymal enlargement, changes in size and echo-texture of the testis and peritesticular hyperemia. In testicular torsion, some epididymal findings such as swelling, enlargement, and heterogenicity, as well as an extra-testicular mass had been de- 
scribed by a few investigators $(7,8)$. However, ultrasound findings of testicular torsion are not pathognomonic, and occasionally, radionuclide imaging and surgery is needed for differentiation.

\section{Objectives}

In this study, we assessed scrotal and epididymal changes in pediatric testicular torsion compared to epididymitis and proposed more specific indicators for differentiation of testicular torsion from epididymitis.

\section{Methods}

From March 2011 to December 2017, a descriptive case - control study was performed in Dr. Sheikh and Akbar Children hospital, Mashhad University of Medical Sciences, Mashhad, Iran by a pediatric radiologist.

Pediatric patients with acute scrotum and suspicious to testicular torsion or epididymitis were examined using multi-frequency surface probes (Esoate, Maylab 50 or class C, $12 \mathrm{MHz}$ ). Sonographic findings of 41 patients (aged 10 days to 12 years) with clinically and surgically proven testicular torsion and epididymitis were documented. Other causes of acute scrotum were excluded from the study.

Our ultrasonic variables were: size, axis, and echotexture of the affected testis, size, shape, location, and echo-pattern of the affected epididymis, the presence of knot or coiling on spermatic cord, hydrocele, scrotal skin thickening, and presence of blood flow in testis and peritesticular surrounding tissues.

We focused mostly on the size, shape, location, and echo-pattern of affected epididymis in testicular torsion and epididymitis cases. A knot or whirlpool of the spermatic cord appeared as an abrupt change in the track of the cord (Figure 1).

The testicular axis was assessed in comparison with cord axis. If long axis of testis was aligned parallel to spermatic cord, it was considered as normal, and if they were perpendiculars to each other, it was considered as abnormal (Figure 2).

The normal epididymis was observed as a comma shaped, the elongated structure located on the posterior border of testis that its head was found at the superior pole of the testis and its tail was found at the inferior pole of the testis attach to testis through mediastinum. Epididymis head was seen as the largest and most recognizable part. In epididymitis, enlargement was usually observed without anatomic displacement and on ultrasound examination of the long axis of the testis, its head and tail were seen in upper and lower poles of the testis. If epididymal head and tail were not seen in upper and lower poles of the testis and aggregated epididymis was observed on mediastinum, it was considered as displaced epididymis. In these cases, aggregation of elongated epididymis in mediastinum had a displaced mass-like configuration of the epididymis (Figure 3).

In epididymitis cases, conservative antibiotic treatment and in testicular torsion patients, appropriate surgical procedures were done. Eventually, the sonographic findings were analyzed to compare the results. Data analysis was performed using SPSS version 16 and P value less than 0.05 was considered as the significance level.

\section{Results}

Out of the 41 patients, 21 cases had testicular torsion, and 20 cases had epididymitis with a similar age range $(P$ $=0.007)$. One case with testicular torsion had an ectopic intra-canal location, and another one had an extra-vaginal type of torsion. Diagnostic value of ultrasonography in the differentiation of torsion from epididymitis using a number of variables is shown in Table 1 .

Table 1. The Diagnostic Power of Numbers of Ultrasonic Variables in the Diagnosi of Torsion ${ }^{\mathrm{a}}$

\begin{tabular}{|lccc|}
\hline Ultrasonic Findings & Sensitivity & Specificity & PValue \\
\hline Testicular heterogeneity & 71 & 94 & 0.000 \\
\hline Testicular axis & 71 & & 0.042 \\
\hline Testicular flow pattern & 95 & 94 & 0.000 \\
\hline $\begin{array}{l}\text { Increased echogenicity of } \\
\text { epididymis }\end{array}$ & 95 & 73 & 0.000 \\
\hline $\begin{array}{l}\text { heterogeneity of epididymis } \\
\begin{array}{l}\text { Abnormal mediastinal } \\
\text { epididymis location }\end{array}\end{array}$ & 100 & 84 & 0.000 \\
\hline $\begin{array}{l}\text { Mass-like configuration of } \\
\text { epididymis }\end{array}$ & 90 & 100 & 0.000 \\
$\begin{array}{l}\text { Geographic or no flow pattern } \\
\text { of epididymis }\end{array}$ & 90 & 100 & 0.000 \\
\hline
\end{tabular}

${ }^{\mathrm{a}}$ Values are expressed as $\mathrm{N}(\%)$.

Testicular enlargement was seen in about 40 of patients with torsion and epididymitis, and the mean of testicular size was not significantly different between them ( $P$ $=0.084)$. Echogenicity of testis was increased in 14.3 and decreased in 28.6 of testicular torsion patients, while it was normal in all epididymitis patients $(\mathrm{P}=0.005)$.

The testicular parenchymal heterogenicity was detected in 71.5 of patients with torsion and 5 of patient with epididymitis ( $\mathrm{P}=0.000$ with 94 specificity). The testicular axis was assessed in comparison with cord axis. If long axis of testis was aligned parallel to spermatic cord, it was 

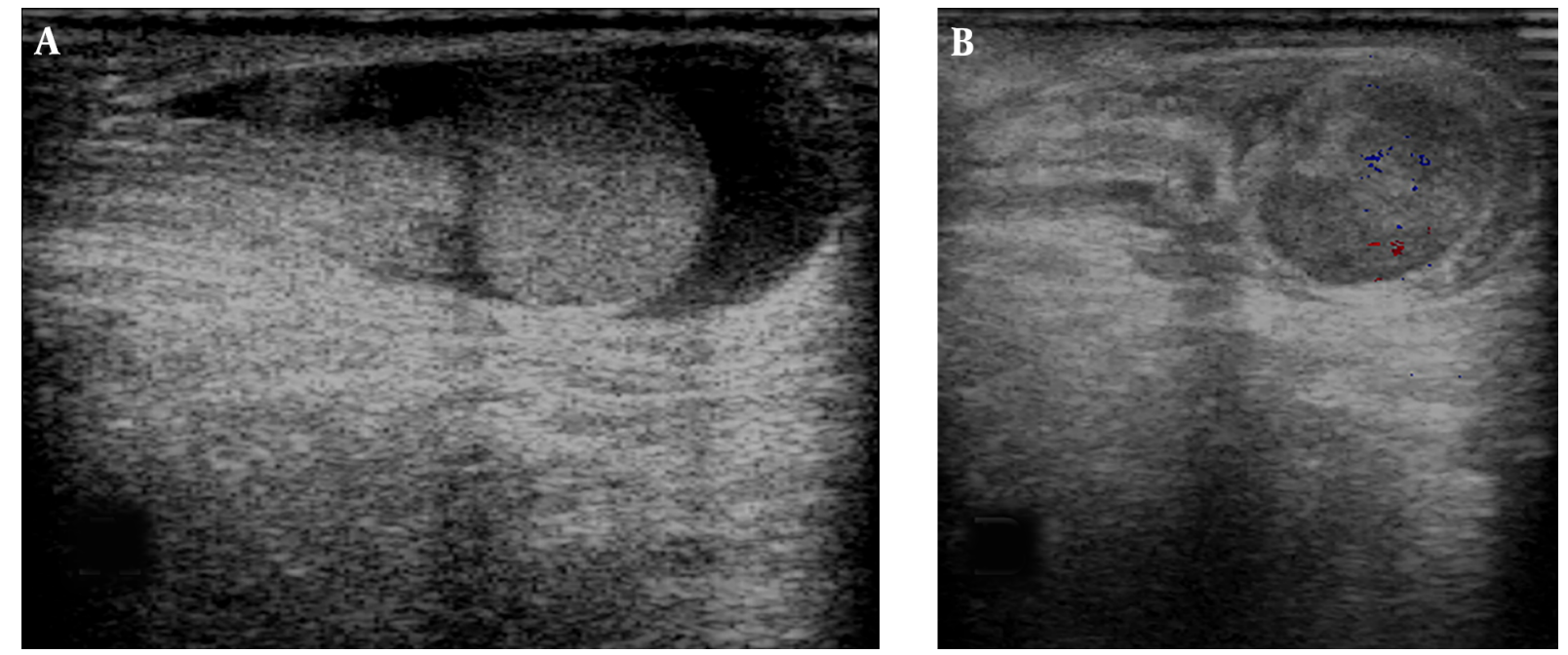

Figure 1. The spermatic cord shape in epididymitis and testicular torsion: In epididymitis case (A), cord enlargement has a straight shape while in B testicular torsion cases (B), it has an abrupt change in the pathway at near to the testis.
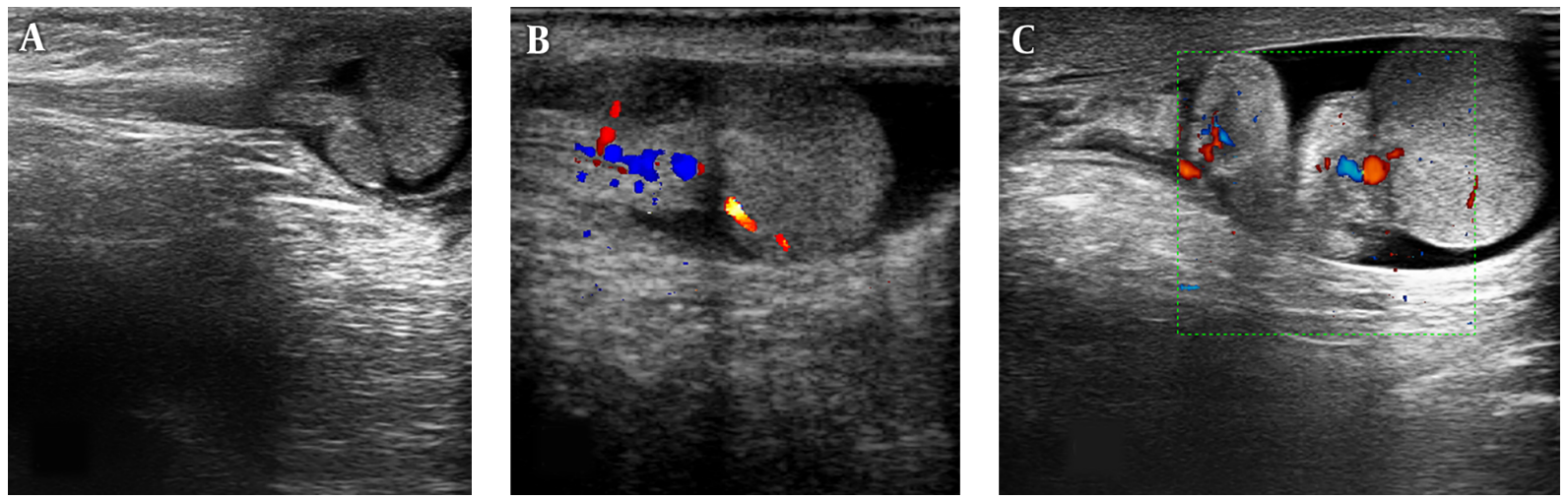

Figure 2. The abnormal orientation (axis) of the testis in normal (A), epididymitis (B) and testicular torsion (C): The spermatic cord axes were aligned with testicular mediastinum and short axis of testis, and not with long axis of testis. The spermatic cord shape at testicular torsion isn't a straight shape and appears as twisted form.

considered as normal, and if they were perpendicular together, it was considered as abnormal. Abnormal testicular axis was seen in both groups (torsion, 71.5 and epididymitis, 35) with significant difference $(\mathrm{P}=0.042)$. Hydrocele was seen in 19 and 30 of patients in both groups without significant difference $(\mathrm{P}=0.292)$.

The testicular flow pattern was increased in 75 of patients with epididymitis and none of the torsion patients, while it was decreased in one case with epididymitis and 95 of patients with torsion $(\mathrm{P}=0.000)$. The hyperemia of surrounding tissues was observed in 95 of torsion cases and 60 of epididymitis cases with significant difference $(P$ $=0.008$ ). The epididymal enlargement was apparent visually in all patients with acute torsion and epididymitis. The scrotal skin thickening was also obvious in all patients of both groups.

A total of 85 patients with epididymitis showed homogenous epididymis echo texture while the 100 patients with torsion showed heterogenic echo epididymis echo texture. The increased echogenicity of epididymis was seen in 95 of testicular torsion patients and 25 the epididymitis patients with significant difference $(\mathrm{P}=0.000)$.

All patients with epididymitis had a normal epididymis location while 90.5 of patients with torsion had an abnormal mediastinal epididymis location. The epididymis mass-like configuration was seen in 76 of patients with torsion and none of epididymitis cases.

There was increased epididymal flow pattern in all 

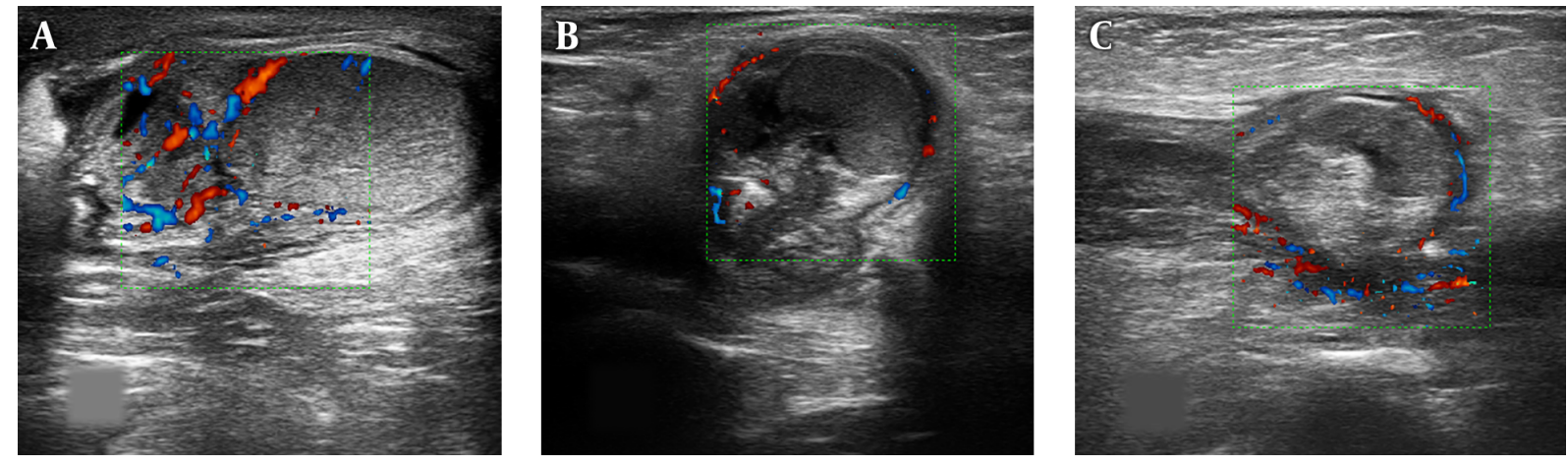

Figure 3. The location of the largest volume of epididymis head in epididymitis and testicular torsion: A) in epididymitis case, epididymis head enlargement without anatomic displacement is seen in the upper pole and lower pole of the testis. B and C) in testicular torsion cases, epididymis isn't seen in the upper pole and lower pole of the testis and aggregate in the mediastinum and forms a displaced mass-like configuration of the epididymis.

cases with epididymitis. Three types of epididymal flow pattern were observed in patients with testicular torsion: uniform flow (9.5), geographic flow (19), and nondetectable flow (71.5). Evaluation of spermatic cord in the torsion group revealed that 43 has straightened, 38 has knotted, and 19 has coiled spermatic cord. In epididymitis group, most patients (83) had a straightened spermatic cord. Difference between two groups was not significant $(P=0.179)$. Using these findings, we haven't missed any case of torsion in this study, and none of our cases with epididymitis underwent unnecessary surgery.

\section{Discussion}

Testicular torsion forms approximately 20 of scrotal emergencies $(1,7)$ and its most common differential diagnosis is epididymitis, which has similar clinical and ultrasonic findings. If the clinical findings suggest torsion with certainty, there is no need for additional imaging, however, if the clinical signs are vague, imaging is needed to make the diagnosis (2). Usually, the vascularity status in Doppler ultrasound is the point of differentiation; absence of testicular blood flow is observed in torsion and increased epididymal and occasionally testicular blood flow is seen in epididymitis. As the detection of testicular blood flow is very difficult or impossible in children (8), others ultrasonic findings are helpful.

Our study shows that testicular and epididymal enlargement, hydrocele, the hyperemia of surrounding tissues, and the scrotal skin thickening are observed in both epididymitis and torsion without any significant difference $(P \geq 0.05)$. Some other findings were observed in both groups with a significant difference $(\mathrm{P} \leq 0.05)$ such as changes in echogenicity of testis and epididymis, abnormal testicular axis and spermatic cord changes are ob- served in both epididymitis and torsion; however, they had low sensitivity. For example, the presence of coiled spermatic cord or knot sign was evident in about half of the cases with testicular torsion in our study and some other researches (9). As non-uniform edematous cord is also seen in epididymitis, it may simulate a knot appearance, thus, it is not a specific sign.

In our study, the most specific signs of testicular torsion were testicular parenchymal heterogenicity (94), testicular flow pattern (94), increased echogenicity of epididymis (73), heterogenicity of epididymis (84), abnormal epididymis location (100), mass-like configuration of epididymis (100), and epididymis flow pattern (100). Epididymis was hyper-vascular and had a normal shape and location in all epididymitis patients. Most of these patients (84) had a homogenous echo texture. Therefore, epididymal findings are more specific and sensitive than testicular signs. A number of these epididymal findings such as swelling, enlargement, and heterogenicity, as well as an extra-testicular mass have been mentioned in a few articles $(4,6,7)$. Nussbaum et al., have reported that epididymal enlargement and hyperechogenicity are detected in 94 and 73 of cases with testicular torsion, respectively (8). Epididymal enlargement was apparent both visually and with measurement $(7,8)$. Hyperechogenicity, heterogeneous echo texture, and enlargement of epididymis may be observed due to venous congestion and some degrees of epididymal necrosis (7).

Changes in shape and location of the epididymis and the epididymis mass-like configuration were the most specific signs of testicular torsion in our study. Bandarkar et al., Galina, and Nussbaum et al., have also reported changes in the shape of epididymis in almost all of their cases (4). Nussbaum et al., explained globular epididymal shape in 87 of patients (8) and Galina et al., reported a complex 
extra-testicular mass (10).

Attention to the location of the epididymal head is a clue to diagnosing displacement. In all of our cases with epididymitis, the epididymal head was present at the upper pole of the testis, while in torsion cases, epididymal head and tail was not seen in poles of the testis and displaced toward mediastinum. Lose attachment of epididymal head and tail to the testis may cause their displacement during torsion.

Avascular or geographic flow patterns of epididymis were observed in 89 of our patients. Absent epididymal blood flow has been observed in 96 in the study of Afsarlar et al., and 93 of cases in Nussbaum et al., researches (7, 8). In contrast, in our study, epididymal hyperemia was obvious in all cases with epididymitis. In epididymitis, the epididymis enlarges as a diffuse hyperemic mass that may involve adjacent testis and causes testicular enlargement and hyper-vascularity.

Although epididymal cystic spaces have been reported in 48 of nonviable cases by Afsarlar et al., (7), however, in our study, they were observed only in two cases with testicular torsion. Epididymal cystic spaces may be vascular channels, dilated efferent ejaculatory, or dilated lymphatic vessels.

Overall, there are several sonographic signs in testis, epididymis, and surrounding tissues for differentiation of torsion from epididymitis, which most of them have a high diagnostic value with the sensitivity of $71-100$ and the specificity of $73-100$. Considering these reliable signs during sonographic examination can minimize the need for further diagnostic approaches.

This study was done in a pediatric hospital and was faced with some limitations. Larger sample size in future studies may improve and extend the results. Also, further researches are helpful if they can compare sonographic results with surgical and pathological findings in operated cases to empower the results and make a better understanding of the subject. Considering the fact that the attach rate of epididymis head and tail to testis is especially important.

\subsection{Conclusions}

Avascularity, heterogenicity, displacement, and masslike configuration of the epididymis are reliable sono- graphic findings for differentiation testicular torsion from epididymitis. They have high diagnostic value with the sensitivity of 75 - 100 and specificity of $84-100$. Therefore, making proper use of them can minimize diagnostic pitfalls.

\section{Acknowledgments}

The authors would like to appreciate pediatric surgeons and pathologists of Akbar Hospital of Mashhad University of Medical Sciences for their advocated help.

\section{References}

1. Yusuf GT, Sidhu PS. A review of ultrasound imaging in scrotal emergencies. J Ultrasound. 2013;16(4):171-8. doi: 10.1007/s40477-013-0033-x. [PubMed: 24432171]. [PubMed Central: PMC3846954].

2. Delaney LR, Karmazyn B. Ultrasound of the pediatric scrotum. Semin Ultrasound CT MR. 2013;34(3):248-56. doi: 10.1053/j.sult.2012.11.010. [PubMed: 23768891].

3. Dogra VS, Rubens DJ, Gottlieb RH, Bhatt S. Torsion and beyond: new twists in spectral Doppler evaluation of the scrotum.JUltrasound Med. 2004;23(8):1077-85. [PubMed: 15284466].

4. Bandarkar AN, Blask AR. Testicular torsion with preserved flow: key sonographic features and value-added approach to diagnosis. Pediatr Radiol. 2018;48(5):735-44. doi: 10.1007/s00247-018-4093-0. [PubMed: 29468365]. [PubMed Central: PMC5895684].

5. Karmazyn B, Steinberg R, Kornreich L, Freud E, Grozovski S, Schwarz $\mathrm{M}$, et al. Clinical and sonographic criteria of acute scrotum in children: a retrospective study of 172 boys. Pediatr Radiol. 2005;35(3):30210. doi: 10.1007/s00247-004-1347-9. [PubMed: 15503003].

6. Prando D. Torsion of the spermatic cord: the main gray-scale and doppler sonographic signs. Abdom Imaging. 2009;34(5):648-61. doi: 10.1007/s00261-008-9449-8. [PubMed: 18709404].

7. Afsarlar CE, Elizondo R, Yilmaz E, Cakmakci E, Ballow DJ, Demir E, et al. Ultrasonographic findings in the epididymis of pediatric patients with testicular torsion. J Pediatr Urol. 2017;13(4):393 e1-6. doi: 10.1016/j.jpurol.2017.05.018. [PubMed: 28713006].

8. Nussbaum Blask AR, Rushton HG. Sonographic appearance of the epididymis in pediatric testicular torsion. AJR Am J Roentgenol. 2006;187(6):1627-35. doi: 10.2214/AJR.05.0461. [PubMed: 17114561].

9. Arce JD, Cortes M, Vargas JC. Sonographic diagnosis of acute spermatic cord torsion. Rotation of the cord: a key to the diagnosis. Pediatr Radiol. 2002;32(7):485-91. doi: 10.1007/s00247-002-0701-z. [PubMed: 12107581].

10. Galina P, Dermentzoglou V, Baltogiannis N, Zarifi M. Sonographic appearances of the epididymis in boys with acute testicular torsion but preserved testicular blood flow on color Doppler. Pediatr Radiol. 2015;45(11):1661-71. doi: 10.1007/s00247-015-3375-z. [PubMed: 26104655]. 